\title{
Topological states of matter: theory and applications
}

\author{
${\text { Roberta } \text { Citro }^{1, a} \text {, Pasquale Marra }}^{2}$, and Francesco Romeo ${ }^{1}$ \\ ${ }^{1}$ Dipartimento di Fisica "E.R. Caianiello", Università degli Studi di Salerno, \\ Via Giovanni Paolo II, 132, 84084 Fisciano (Sa), Italy \\ 2 RIKEN, Center for Emergent Matter Science, Wakoshi, Saitama 351-0198, Japan
}

Received 15 November 2018

Published online 18 December 2018

This volume is a collection of papers devoted to topological states of matter in various fields of physics ranging from condensed matter to photonic systems, and their application in quantum technologies. The volume aims at illustrating the actual stateof-the-art and emerging challenges for topological systems. Peculiar aspects of zero(quantum dots), quasi-one- (quantum Hall edge states, quantum wires, and ladders), and three-dimensional systems (crystalline insulators, hybrid junctions) are analyzed. The volume also includes the discussion of relevant aspects about photonic systems that make them an ideal playground for the rapidly growing field of non-Hermitian models.

Nowadays, topological states of matter are believed to be a crucial ingredient to design a quantum computer. Implementing a full-scale quantum computer is a major challenge of modern physics. A promising route to achieve fault-tolerant quantum computation is the engineering and control of topological states of matter, which may lead to the realization of a topological qubit. In this regard, the role of Majorana bound states is of fundamental importance: these topologically protected quasiparticles hosted by topological superconductors have been proposed as the basis of a topological quantum computer due to their non-Abelian braiding statistics. In particular, the braiding of Majorana bound states can be implemented to perform fault-tolerant quantum operations.

In this volume,various aspects of Majorana bound states are discussed, e.g., their experimental evidence and their robustness with respect to perturbations.

For example, recently, the quest for topological states of matter has turned to non-Hermitian systems, which exhibit a rich variety of unique properties that have no Hermitian counterparts. Lattices modeled through non-Hermitian Hamiltonians appear in the context of photonic systems, where one needs to account for gain and loss, circuits of resonators, or in the context of condensed matter systems, where they take into account the finite lifetime of electronic states due to interactions or in systems out of equilibrium. Alvares et al. [1] provide a brief review of this rapidly growing subject, the search for topological states and a bulk-boundary correspondence in non-Hermitian systems.

Lau and Ortix [2] in their mini-review discuss recent advances in the study of topological insulators protected by spatial symmetries by reviewing three representative examples. In three dimensions, topological states of matter are generally characterized

a e-mail: citro@sa.infn.it 
by the presence of gapless boundary states at surfaces that respect the protecting spatial symmetry. The authors discuss the appearance of these topological states both in crystals, with negligible spin-orbit coupling, and a four-fold rotational symmetry, as well as in mirror-symmetric crystals with sizable spin-orbit interaction, characterized by the so-called mirror Chern number. Finally, they discuss similar topological crystalline states in one-dimensional insulators, such as nanowires or atomic chains, with mirror symmetry. There, the prime physical consequence of the nontrivial topology is the presence of quantized end charges.

A very promising field of physics, where topological states of matter play an important role, is the electron-based counterpart of quantum optics, which requires the capability to generate and control single electron wave packets. The edge states of quantum spin Hall systems, i.e., two-dimensional topological insulators realized in $\mathrm{HgTe} / \mathrm{CdTe}$ and InAs/GaSb quantum wells, may turn the tide in the field, as they do not require the magnetic field that limits the implementations based on quantum Hall effect. However, the band structure of these topological states, described by a massless Dirac fermion Hamiltonian, prevents electron photoexcitation via the customary vertical electric dipole transitions of conventional optoelectronics. So far, proposals to overcome this problem are based on magnetic dipole transitions induced via Zeeman coupling by circularly polarized radiation, and are limited by the $g$-factor. Alternatively, optical transitions can be induced from the edge states to the bulk states, which are not topologically protected though. In their review, Dolcini and Rossi [3] show that an electric pulse, localized in space and/or time and applied at a quantum spin Hall edge state, can photoexcite electron wavepackets by intra-branch electrical transitions, without invoking the bulk states or the Zeeman coupling. Such wavepackets are spin-polarized and propagate in opposite directions, with a density profile that is independent of the initial equilibrium temperature and that does not exhibit dispersion, as a result of the linearity of the spectrum and of the chiral anomaly characterizing massless Dirac electrons. They further investigate the photoexcited energy distribution and show how, under appropriate circumstances, minimal excitations (levitons) are generated. Furthermore, they also show that the presence of a Rashba spin-orbit coupling can be exploited to tailor the shape of photoexcited wavepackets.

Along the same line, Ferraro et al. [4] instead review and develop recent results regarding leviton excitations generated in topological states of matter - such as integer and fractional quantum Hall edge channels - and carrying a charge that is a multiple of the elementary charge. The peculiar features associated to these clean and robust emerging excitations can be detected through current correlation measurements. In particular, relevant information can be extracted from the noise signal in generalized Hong-Ou-Mandel experiments, where levitons with different charges collide against each other at a quantum point contact. They describe this quantity both in the framework of the photo-assisted noise formalism and in terms of a very interesting and transparent picture based on wave-packet overlap.

Bercioux and Lucignano [5] in their contribution investigate the thermoelectric properties of a hybrid junction realized coupling surface states of a three-dimensional topological insulator with a conventional $s$-wave superconductor. They focus on the ballistic devices and study the quasiparticle flow, carrying both electric and thermal currents, adopting a scattering matrix approach based on conventional BlonderTinkham-Klapwijk formalism. They calculate the cooling efficiency of the junction as a function of the microscopic parameters of the normal region (e.g., the chemical potential). The cooling power increases when moving from a regime of Andreev specular-reflection to a regime where Andreev retro-reflection dominates. Differently from the case of a conventional N/S interface, they can achieve efficient cooling of the normal region, without including any explicit scattering potential at the interface, to increase normal reflection. 
Fleckenstein et al. [6] discuss a very interesting interface phenomenon. The presence of a Majorana bound state in condensed matter systems is often associated to a zero-bias peak in conductance measurements. Here, they analyze a system where this paradigm is violated: A Majorana bound state is always present at the interface between a quantum spin Hall system that is magnetically gapped and a quantum spin Hall system gapped by proximity induced $s$-wave superconductivity. However, the linear conductance could be either zero or nonzero and quantized depending on the energy and length scales of the barriers. The transition between the two values is reminiscent of the topological phase transition in proximized spin-orbit coupled quantum wires in the presence of an applied magnetic field.

Cayao and Black-Schaffer [7] numerically analyze the effect of finite length of the superconducting regions on the low-energy spectrum, current-phase curves, and critical currents in junctions between trivial and topological superconductors. Such junctions are assumed to arise in nanowires with strong spin-orbit coupling under external magnetic fields and proximity-induced superconductivity. They show that all these quantities exhibit a strong dependence on the length of the topological sector in the topological phase and serve as indicators of the topological phase, which is established when Majorana bound states appear at the end of the topological superconductor.

Going from one- to two-dimensions, the simplest generalization of a single topological chain is the Kitaev ladder. In their paper, Maiellaro et al. [8] discuss the topological phase diagram of a Kitaev ladder, where a full understanding of the effect of the couplings between the chains is still missing. The system studied shows a richer phase diagram where the coupling between the two chains gives rise to a direct transition from a state with two to zero energy Majorana bound states per each side, while for large interchain hopping, the system behaves as an effective single Kitaev chain with only one zero energy-bound state per side.

In their manuscript, Feng and Wang propose [9] to directly measure the Majorana number for one-dimensional topological superconductors using a quantum dot. The setup consists of two topological superconducting wires with four Majorana zero modes, which are coupled to an external quantum dot. The measurement is achieved by utilizing the definition of the Majorana number, which is the charge-parity flipping when changing the boundary condition for the topological superconductor. They consider a control of the boundary condition with voltage gates: When the voltage on the gate is modulated sequentially, the boundary conditions change and the parity of the superconducting state flips. They demonstrate that this parity flipping will change the electron occupation probability of the quantum dot, which reflects the value of the Majorana number.

Of central importance for the applications in topological quantum computation is the anionic statistics, which is expected to be realized by Majorana bound states. In his contribution, Kashuba [10] addresses the transport correlations in interacting systems in the presence of Majorana bound states, specifically in a spinful topological Kondo model connected to a topological one-dimensional superconductor. It is shown how the analysis of cross-correlations at high energy can identify the presence of the topologically nontrivial states and probe their anionic statistics. The proposal is relevant to experiments on superconducting nanowires with strong spin-orbit coupling, and may shed light on the possibility to realize the braiding procedures necessary for topological computations.

We really hope that this volume can contribute in evidencing new challenges and trends in the exciting field of topological matter and stimulate further results in the field. 


\section{References}

1. V.M. Martinez Alvarez, J.E. Barrios Vargas, M. Berdakin, L.E.F. Foa Torres, Eur. Phys. J. Special Topics 227, 1295 (2018)

2. A. Lau, C. Ortix, Eur. Phys. J. Special Topics 227, 1309 (2018)

3. F. Dolcini, F. Rossi, Eur. Phys. J. Special Topics 227, 1323 (2018)

4. D. Ferraro, F. Ronetti, L. Vannucci, M. Acciai, J. Rech, T. Jockheere, T. Martin, M. Sassetti, Eur. Phys. J. Special Topics 227, 1345 (2018)

5. D. Bercioux, P. Lucignano, Eur. Phys. J. Special Topics 227, 1361 (2018)

6. Ch. Fleckenstein, F. Keidel, B. Trauzettel, N. Traverso Ziani, Eur. Phys. J. Special Topics 227, 1377 (2018)

7. J. Cayao, A.M. Black-Schaffer, Eur. Phys. J. Special Topics 227, 1387 (2018)

8. A. Maiellaro, F. Romeo, R. Citro, Eur. Phys. J. Special Topics 227, 1397 (2018)

9. J.-J. Feng, Z. Wang, Eur. Phys. J. Special Topics 227, 1405 (2018)

10. O. Kashuba, Eur. Phys. J. Special Topics 227, 1413 (2018) 